EPJ Web of Conferences 70, 00054 (2014)

DOI: 10.1051/epjconf/20147000054

(C) Owned by the authors, published by EDP Sciences, 2014

\title{
EPR measurement and the origin of cosmic density fluctuations
}

\author{
Masahiro Morikawa ${ }^{1, a}$ \\ Department of Physics, Ochanomizu University, 2-1-1 Otsuka, Bunkyo, Tokyo 112-8610, Japan \\ ${ }^{1}$ Department of Physics, Ochanomizu University, 2-1-1 Otsuka, Bunkyo, Tokyo 112-8610, Japan
}

\begin{abstract}
We explore consistent application of quantum mechanics to the objects in the Universe and in laboratories. The measurement dynamics in quantum mechanics is modeled as a physical process of spontaneous symmetry breaking (SSB) which is described by the generalized effective action method. A violation of the Bell inequality is observed in this model and the generation of the density fluctuations in the early Universe is described as the SSB process of the spatially translational symmetry.
\end{abstract}

\section{Introduction}

Quantum mechanics is an excellent theory to describe the Universe. For example, the mass and the radius of typical planets are given by

$$
M=\frac{e^{3} k_{0}^{3 / 2}}{2 \sqrt{2} G^{3 / 2} m_{p}^{2}} \approx 8.1 \times 10^{26} \mathrm{Kg}, \quad R=\frac{\hbar^{2}}{3 \sqrt{2 G k_{0}} e m_{e} m_{p}} \approx 1.0 \times 10^{7} \mathrm{~m}
$$

where $m_{e}$ and $m_{p}$ are the electron and proton masses, respectively. These expressions are derived by minimizing the energy $E=p^{2} /\left(2 m_{e}\right)-k_{0} e^{2} / x$ of an electron in the Hydrogen atom using the uncertainty principle $(\Delta x)(\Delta p) \approx \hbar / 2$. Moreover, the scale of typical stars, which are in equilibrium with the nuclear fusion process, is given by the balance of the energy uncertainty $E \approx p^{2} /\left(2 m_{p}\right)$ and the Coulomb barrier $k_{0} e^{2} / x$. This balance yields the approximate temperature $T \approx 5 \times 10^{8} \mathrm{~K}$. Further balance of the gravitational energy and the Coulomb energy in the whole star yields the expression

$$
M=\frac{e^{3} k_{0}^{3 / 2}}{8 G^{3 / 2} m_{e}^{3 / 2} m_{p}^{1 / 2}} \approx 2.3 \times 10^{31} \mathrm{Kg}, \quad R=\frac{39.5 \sqrt{e V} \hbar^{3}}{e^{3} \sqrt{G} k_{0}^{3 / 2} m_{e}^{3 / 2} m_{p}} \approx 3.2 \times 10^{8} \mathrm{~m}
$$

for a typical star. The appearance of $\hbar$ in the above radii $R$ becomes more evident in the degenerate stars, in which both the mass and the radius are proportional to $\hbar^{3 / 2}$. These observations indicate the fact that quantum mechanics is indispensable in the Universe. There is yet another implication in the case of bosons [1]. If some kind of light boson field $(m \leq 20 \mathrm{eV})$ dominates the Universe as dark matter, then it can yield Bose-Einstein condensation (BEC) which behaves as a classical scalar field $\varphi(x)$. This condensed field successfully describes the dynamics of dark energy [1]. Quantum mechanics is thus fully compatible with astrophysics in the Universe.

${ }^{\mathrm{a}}$ hiro@phys.ocha.ac.jp

This is an Open Access article distributed under the terms of the Creative Commons Attribution License 2.0, which permits unrestricted use, distribution, and reproduction in any medium, provided the original work is properly cited. 
However, if we consider the operational description of quantum mechanics, then the whole argument becomes quite complicated. We cannot describe the collapse of the wave function of the Universe since no explicit observer is there. The whole structure in the Universe might fall into big illusion.

More serious problem arises when we describe the origin of the density fluctuations from quantum mechanics. In the inflationary era in the early Universe, the k-mode of the light scalar field behaves as $v_{k}^{\prime \prime}+\left(k^{2}-2 H^{2} e^{2 H t}\right) v_{k}=0$, whose solution in de Sitter invariant vacuum behaves as $v_{k} \approx k^{-1 / 2}\left(1+i k^{-1} H e^{H t}\right)$. A standard calculation for the power spectrum $P(k)$ implicitly assumes the identification of the quantum correlation of the scalar field $\langle\hat{\phi}(x) \hat{\phi}(y)\rangle_{k}$ and the statistical fluctuation $|\delta \varphi(k)|^{2}$ [2] [3]. The latter violates the spatial translational invariance while the former does not. This symmetry breaking of the translational invariance is indispensable for the generation of classical density fluctuations [4] [5].

It is important to notice that the origin of the problem is common with the laboratory quantum mechanics. The problem stems from the lack of physical description of the quantum measurement apparatus, which should have autonomous dynamics independent from an artificial operator. Therefore we first consider a physical dynamics of the quantum measurement process and then apply it to the laboratory measurement and to the early Universe.

\section{Physics of quantum measurement}

Our proposal is that the measurement is a spontaneous symmetry breaking (SSB) process and is described by the generalized effective action method [6] [7]. The classical order parameter of the field which cause SSB corresponds to the meter of the measurement apparatus. The generalized effective action fully describes the dynamics of this field and the order parameter. This field couples to the system and affects the evolution of the density matrix of the system. The finite filed $\varphi=\langle\hat{\phi}\rangle \neq 0$ means the state of the field $\hat{\phi}$ is a coherent state or BEC state. The macroscopic condensation guarantees the classical property of the field $\varphi$.

A key observation how the generalized effective action describes the SSB is to consider the entropy $S_{e}=-k_{B} \operatorname{Tr}(\rho \ln \rho)$, and the action $S_{a}=-i \hbar \ln \Phi$, where $\rho=|\Phi\rangle\langle\Phi|$. Then the entropy becomes

$$
S_{e}=\frac{k_{B}}{\hbar} \operatorname{Im}\left[\sum\left(S_{a}-S_{a}^{*(\text { time reversal })}\right)\right]
$$

where the quantity $\sum\left(S_{a}-S_{a}^{*(\text { time reversal) }}\right)$ in the right hand side is almost the generalized effective action $\tilde{\Gamma}$. This observation suggests that $\operatorname{Im} \tilde{\Gamma}$ describes statistical property of the system while $\operatorname{Re} \tilde{\Gamma}$ describes the ordinary dynamics.

There is a neat formalism of the generalized effective action method [6] [7]. We simply review the essence of it in the case of real scale field. The effective action $\tilde{\Gamma}$ is a functional of the classical field $\varphi_{c} \equiv\left(\varphi_{+}+\varphi_{-}\right) / 2$ and $\varphi_{\Delta} \equiv \varphi_{+}-\varphi_{-}: \tilde{\Gamma}\left[\varphi_{c}, \varphi_{\Delta}\right]$, where the support of $\varphi_{+}$is the ordinary time contour $-\infty \rightarrow \infty$ while that of $\varphi_{-}$is the reversed time contour $\infty \rightarrow-\infty$. The imaginary part is generally even in $\varphi_{\Delta}$ and within the Gaussian approximation,

$$
\operatorname{Im} \tilde{\Gamma}=\frac{1}{2} \iint \varphi_{\Delta}(x) B(x-y) \varphi_{\Delta}(y)+\ldots
$$

where the kernel $B(x-y)$ is positive definite. Therefore by introducing real auxiliary field $\xi(x)$, we have

$$
\exp (i \tilde{\Gamma})=\int[d \xi] P[\xi] \exp \left[i \operatorname{Re} \tilde{\Gamma}+i \int \xi \varphi_{\Delta}\right]
$$


where $P[\xi] \equiv \exp \left[-\frac{1}{2} \iint \xi B^{-1} \xi\right]$ can be interpreted as statistical weight function. On the other hand $\operatorname{Re} \tilde{\Gamma}+\xi \varphi_{\Delta} \equiv S_{\text {eff }}$ is real and we can apply the least action principle as usual $\delta S_{\text {eff }} /\left.\delta \varphi_{\Delta}(x)\right|_{\varphi_{\Delta}=0}=-J_{c}$. This yields a probabilistic differential equation, which is real and causal:

$$
\left(\square+m^{2}\right) \varphi_{c}(x)=-V^{\prime}+\int_{-\infty}^{t} d t^{\prime} \int d^{3} x^{\prime} A\left(x-x^{\prime}\right) \varphi_{c}\left(x^{\prime}\right)+\xi(x),
$$

where $A\left(x-x^{\prime}\right)$ represents retarded kernel. The stochastic force term $\xi(x)$, which arose from $\operatorname{Im} \tilde{\Gamma}$, triggers SSB and the classical field $\varphi_{c}(x)$ eventually controls the measurement process as we will see soon.

The most simple model of spin (1/2) measurement by the field $\phi$ [8] will be given by the Lagrangian.

$$
L=\frac{1}{2}\left(\partial_{\mu} \phi\right)^{2}-\frac{m^{2}}{2} \phi^{2}-\frac{\lambda}{4 !} \phi^{4}+\mu \phi \vec{S} \cdot \vec{B}+(\text { bath }),
$$

where $m^{2}<0, \lambda>0, \mu>0$. From the method of generalized effective action, we have a set of equations, for the order parameter or the meter of the apparatus,

$$
\frac{d \varphi}{d t}=\gamma \varphi-\frac{\lambda}{3 !} \varphi^{3}+\mu\langle\vec{S}\rangle \cdot \vec{B}+\xi
$$

where we set $\left\langle\xi(t) \xi\left(t^{\prime}\right)\right\rangle=\varepsilon \delta\left(t-t^{\prime}\right)$ for simplicity, and for the density matrix of the system,

$$
\frac{d \rho}{d t}=-i \omega\left[S_{3}, \rho\right]+a\left[S_{+} \rho, S_{-}\right]+b\left[S_{-} \rho, S_{+}\right]+c\left[S_{3} \rho, S_{3}\right]+\text { h.c. }
$$

where $a, b$, and $c$ are the bath-correlation functions including the order parameter $\varphi$, and $a=$ $\exp (-\hbar \mu \varphi(t) B /(k T)) b$. If initially $\langle\vec{S}\rangle \cdot \vec{B}>0$, then the linear bias forces $\varphi$ to move toward $\varphi_{+}>0$. Then the effective temperature reduces $k T /(\mu \varphi(t)) \rightarrow 0$, and the spin falls into the corresponding ground state and becomes pure up-state. This further makes initial $\langle\vec{S}\rangle \cdot \vec{B}$ increase. If initially $\langle\vec{S}\rangle \cdot \vec{B}>0$, then the opposite situation makes the spin purely down-state with $\varphi \rightarrow \varphi_{-}<0$. This positive feedback is the essence of the quantum measurement. Eq.(8) describes the process of SSB violating the original symmetry $\phi \rightarrow-\phi$ in the Lagrangian Eq.(7). This method predicts the finite time scale of the measurement $t_{0}=(2 \gamma)^{-1} \ln \left[(g / \gamma)\left(\delta^{2}+(\varepsilon / \gamma)\right)\right]^{-1}$, where $\delta \equiv(\mu / \gamma)\langle\vec{S}\rangle \cdot \vec{B}$.

\section{Quantum mechanics in laboratories}

We now apply this model to the EPR measurement of two spins $\vec{S}_{1}, \vec{S}_{2}$ [9]. We set spatially separated local measurement apparatus composed from the fields $\phi_{1}, \phi_{2}$ for spins $\vec{S}_{1}, \vec{S}_{2}$ respectively, with the same Lagrangian for each set. We are especially interested in the maximally entangled initial state of spins $|\psi\rangle=\left(|\uparrow\rangle_{1} \otimes|\downarrow\rangle_{2}-|\downarrow\rangle_{1} \otimes|\uparrow\rangle_{2}\right) / \sqrt{2}$. We have the evolution equation of the order parameters or the meters of the measurement apparatus $\varphi_{1}$ and $\varphi_{2}$ :

$$
\begin{aligned}
& \dot{\varphi}_{1}=\gamma \varphi_{1}-\frac{\lambda}{3 !} \varphi_{1}^{3}+\mu \operatorname{Tr}\left[\rho \vec{S}_{1} \cdot \vec{B}_{1}\right]+\vec{\xi}_{1} \cdot \vec{B}_{1}+\vec{\xi}_{1}^{(0)}, \\
& \dot{\varphi}_{2}=\gamma \varphi_{2}-\frac{\lambda}{3 !} \varphi_{2}^{3}+\mu \operatorname{Tr}\left[\rho \vec{S}_{2} \cdot \vec{B}_{2}\right]+\vec{\xi}_{2} \cdot \vec{B}_{2}+\vec{\xi}_{2}^{(0)},
\end{aligned}
$$

where the imaginary part of the effective action yields the statistical correlation for the classical stochastic forces: $\left\langle\xi_{1 i} \xi_{2 j}\right\rangle=\operatorname{Tr}\left(\rho(t) S_{i}^{(1)} S_{j}^{(2)}\right)$. The fields $\xi_{1}^{(0)}$ and $\xi_{2}^{(0)}$ are the previous stochastic 
forces arising from the bath for each apparatus. If the initial state is the above most entangle state $|\psi\rangle$, then we have the complete anti-correlation: $\left\langle\xi_{1 i} \xi_{2 j}\right\rangle=-\delta_{i j}$. For the system state $\rho(t) \equiv \rho_{1} \otimes \rho_{2}$, the same equation $(9)$ holds. This set of equations $(9,10)$ determines the full quantum mechanical time evolution including the measurement process as previously.

We can observe a violation of Bell-inequality in this formalism. The probability that the detector apparatus- 1 reads the spin-up state under the applied magnetic field $\vec{B}_{1}$ becomes [8]

$$
P_{+}^{(1)}\left(\vec{B}_{1}\right)=\int_{0}^{\infty} P\left(\varphi_{1}, t\right) d \varphi=\frac{1+\operatorname{erf}(\delta / \sqrt{2 \varepsilon(t)})}{2}
$$

where $P\left(\varphi_{1}, t\right)$ is the probability distribution function of the order parameter $\varphi_{1}$ at time $\mathrm{t}$. The expression in the most right hand side is given by the approximate solution of the Fokker-Planck equation corresponding to $(10)$. In the above, $\delta=\vec{\xi}_{1} \cdot \vec{B}_{1} / \gamma, \sqrt{2 \varepsilon(t)} \approx \gamma^{-1 / 2}$. We also have the similar expression for $P_{-}^{(1)}\left(\vec{B}_{1}\right)$ and $P_{ \pm}^{(2)}\left(\vec{B}_{2}\right)$. Defining

$$
P_{++} \equiv\left\langle P_{+}^{(1)}\left(\vec{B}_{1}\right) P_{+}^{(2)}\left(\vec{B}_{2}\right)\right\rangle, P_{+-} \equiv\left\langle P_{+}^{(1)}\left(\vec{B}_{1}\right) P_{-}^{(2)}\left(\vec{B}_{2}\right)\right\rangle, \ldots
$$

and arranging the magnetic field strengths as $\left|\vec{B}_{1}\right|\left|\vec{B}_{2}\right|=\gamma$, we have

$$
\begin{aligned}
C\left(\vec{B}_{1}, \vec{B}_{2}\right) & =P_{++}+P_{--}-\left(P_{+-}+P_{-+}\right) \\
& =\left\langle\operatorname{erf}\left(\gamma^{-1 / 2} \vec{\xi}_{1} \cdot \vec{B}_{1}\right) \operatorname{erf}\left(\gamma^{-1 / 2} \vec{\xi}_{2} \cdot \vec{B}_{2}\right)\right\rangle \\
& \approx-\gamma^{-1} \vec{B}_{1} \cdot \vec{B}_{2}=-\cos \theta_{12}
\end{aligned}
$$

where $\theta_{12}$ is the angle between the directions of $\vec{B}_{1}$ and $\vec{B}_{2}$. For some range of the configuration of $\vec{B}_{1}, \vec{B}_{2}, \vec{B}_{2}^{\prime}$ and $\vec{B}_{2}^{\prime}$, we have the relation

$$
\left|C\left(\vec{B}_{1}, \vec{B}_{2}\right)+C\left(\vec{B}_{1}^{\prime}, \vec{B}_{2}\right)+C\left(\vec{B}_{1}, \vec{B}_{2}^{\prime}\right)-C\left(\vec{B}_{1}^{\prime}, \vec{B}_{2}^{\prime}\right)\right|>2
$$

which shows a violation of Bell-inequality. Thus the present formalism does not belong to the category of hidden variable theories, but being just another description of quantum mechanics.

\section{Quantum mechanics in the Universe}

In the case of early Universe, the generation process of the primordial density fluctuations is very similar to the above EPR measurement. In the inflationary model, a scalar field, called inflaton, is introduced to yield exponential cosmic expansion: $a(t) \propto e^{H t}$, with $H$ is almost constant. In this space-time, the scalar field fundamental mode $v_{k}(t)$ obeys $v_{k}^{\prime \prime}+\left(k^{2}-2 H^{2} e^{2 H t}\right) v_{k}=0$. This solves as $v_{k} \approx k^{-1 / 2}\left(1+i k^{-1} H e^{H t}\right)$, which represents a strongly squeezed state.

In the standard cosmological scenario, one simply adopts the identification of the quantum correlation $\left.\langle\hat{\phi}(x) \hat{\phi}(y)\rangle\right|_{k}$ as statistical fluctuation $|\delta \varphi(k)|^{2}$ assuming that the fluctuation modes become classical when they cross the horizon. Then, the power spectrum is given by $P_{\delta \varphi} \approx(H /(2 \pi))^{2}$ at the horizon crossing time. This is a scale invariant spectrum which simply reflects the fact that all the modes evolve analogously in de Sitter space.

However actually, the above squeezed state is in the quantum state which still respects the spatial translational invariance. If there were no physical measuring process or a spontaneous symmetry breaking process, never arises the classical fluctuation pattern which violates the symmetry. The 
most promising process for this role would be the nonlinear interaction at the reheating era just after the inflation. In the simple $\lambda \phi^{4}$ model, the k-mode mean field $\varphi_{k}$ acts as an order parameter of the measuring apparatus.

The generalized effective action

$$
\tilde{\Gamma}=\tilde{\Gamma}_{0}+\int \lambda \tilde{\varphi}_{0}^{2} \delta \tilde{\varphi}(k)\langle\tilde{\phi}(x) \tilde{\phi}(y)\rangle \lambda \tilde{\varphi}_{0}^{2} \tilde{\varphi}(-k) d k+\ldots
$$

has the real kernel

$$
\operatorname{Re}\langle\phi(\vec{z}) \phi(0)\rangle=\int_{0}^{\infty} d k \frac{\sin [k z]}{4 \pi^{2} z}\left[\left(\frac{\eta^{\prime 3}-\eta^{3}}{3 \eta^{\prime} \eta}\right)+O\left(k^{3}\right)\right] \equiv G_{\mathrm{ret}}
$$

and the imaginary kernel

$$
\operatorname{Im}\langle\phi(\vec{z}) \phi(0)\rangle=\int_{0}^{\infty} d k \frac{\sin [k z]}{4 \pi^{2} z}\left[\begin{array}{l}
\frac{-1}{\eta^{\prime 2}}-\frac{1}{2}\left(\frac{\eta}{\eta^{\prime}}+\frac{\eta^{\prime}}{\eta}\right) \\
+O\left(k^{2}\right)
\end{array}\right] \equiv G_{\mathrm{c}},
$$

where $\eta \equiv-1 /(a H)$. The real kernel is infrared finite and yields finite renormalization contribution disregarding the ultraviolet manipulations. On the other hand, the imaginary kernel that yields statistical correlation is infrared divergent. However this does not violate the physical evolution of the order parameter since the stochastic force is always finite at each moment. Contrary to the laboratory case, we did not consider the system state because the measurement process now is an absorptive type and the system quanta is lost after the interaction. The relevant information in the system is, however, inherited by the order parameter $\varphi(x)$.

Applying the least action principle on $\operatorname{Re} \tilde{\Gamma}+\xi \varphi_{\Delta} \equiv S_{\text {eff }}$, we have

$$
\ddot{\varphi}(x)+V^{\prime}(\varphi(x))+\int_{-\infty}^{t_{x}} d y G_{\text {ret }}(x-y) \varphi(y)=\xi(x),
$$

where the real part of the effective action yields the infrared-finite renormalization. The stochastic force $\xi(x)$ has the correlation $\langle\xi(x) \xi(y)\rangle=G_{\mathrm{c}}(x-y) \lambda^{2} \varphi_{0}(x)^{2} \varphi_{0}(y)^{2}$ derived from the imaginary part of the generalized effective action. Since the most relevant part of the above equation becomes $\ddot{\varphi}_{k} \approx \xi_{k}$, we have

$$
P_{\delta \varphi}=\frac{4 \pi k^{3}}{(2 \pi)^{3}}\left|\varphi_{k}\right|^{2} \underset{k /(a H)=1}{\longrightarrow} \lambda^{2}\left(\Delta t \varphi_{0}\right)^{4}\left(\frac{H}{2 \pi}\right)^{2},
$$

where $\Delta t$ is the time scale of the reheating era and $\varphi_{0}$ is the typical amplitude of the order parameter in this era.

In the present $\lambda \phi^{4}$ case at the reheating era, $\Delta t \approx \varphi_{0} / \dot{\varphi}_{0}$, and $V\left(\varphi_{0}\right) \approx 0$ yield

$$
P_{\delta \varphi}=O(1)\left(\frac{H}{2 \pi}\right)^{2}
$$

which is almost the same as the standard result. In this special case, large amplitude of the order parameter $\varphi_{0}$ exactly cancels the small time scale $\Delta t$ to yield the factor of order one in front of the scale free power spectrum. On the other hand for example in the chaotic inflationary model, small field $\varphi_{0} \approx 0$ at reheating may yield very small fluctuations. Although the scale free property of the power spectrum is quite robust, the amplitude generally depends on the interaction at reheating. 


\section{Conclusions}

Quantum measurement process is physical. Actually we constructed a simple measurement model in which all the time evolution including the measurement process is autonomously described by the set of Eqs. $(8,9)$. This model is based on the spontaneous symmetry breaking (SSB) in the apparatus. On the other hand in the system, initially entangled system with the apparatus eventually decohere and then procohere with firm correlation between the system and the order parameter.

This method is applied to EPR measurement of spins in laboratories and the effective action method correctly yields the correlation of the two detectors. This correlation is fully consistent with quantum mechanics and the violation of Bell inequality is observed. The essence of the process here is the SSB among the two equivalent detectors, as well as SSB within each detector.

On the other hand in the early Universe, the very similar process could spontaneously break the spatial translational symmetry to yield density fluctuations as classical statistical object. In this case, the SSB process at the reheating when the nonlinearity enters is essential and this nonlinearity determines the strength of the density fluctuations while the scaling in the power spectrum is unchanged.

This symmetry breaking of the spatial translation is indispensable for the generation of the classical density fluctuations in the early Universe. Popular quantum decoherence process itself does not break this symmetry. The quantum mode which goes outside the horizon does not break this symmetry. In the present paper, the SSB of this symmetry was successfully described by the generalized effective action method with its imaginary component due to the squeezing of the state and the nonlinearity through the reheating process.

\section{References}

[1] T. Fukuyama and M. Morikawa, Prog. Theor. Phys. 1151047 (2006); Phys.Rev.D80:063520 (2009).

[2] T. Padmanabhan, Structure Formation in the Universe, Cambridge University Press (1993).

[3] S. Weinberg, Cosmology, Oxford University Press (2008).

[4] M. Morikawa, Prog. Theor. Phys. 771163 (1987).

[5] G. Leon et al, arXiv:1107.3054v1 (2011).

[6] M. Morikawa, Phys. Rev. D33 3607 (1986).

[7] M. Morikawa, Prog. Theor. Phys. 93, 685 (1995).

[8] M. Morikawa and A. Nakamichi, Prog. Theor. Phys. 116, 679 (2006).

[9] A. Einstein, B. Podolsky, and N. Rosen, Phys. Rev. 47777 (1935). 